\title{
Tunica Adventitia
}

National Cancer Institute

\section{Source}

National Cancer Institute. Tunica Adventitia. NCI Thesaurus. Code C33818.

The outer connective tissue layer of the blood vessel wall. The adventitia consists of longitudinally arrang ed collagen fibers, elastic fibers, smooth muscle, fibroblasts, nerves and the vasa vasorum. It is thicker in veins than in arteries. 